\title{
Comparison Study on Development Path for Small and Medium-sized Enterprises E-commerce Using Complex Fuzzy Sets
}

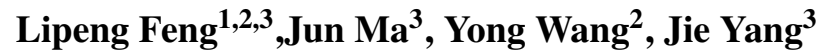 \\ ${ }^{1}$ School of Economics and Management, Chongqing University of Arts and Sciences, \\ Yongchuan, Chongqing 402160, China, \\ E-mail:1078820116@qq.com \\ ${ }^{2}$ School of Economics and Business Administration, Chongqing University, \\ Shapingba, Chongqing, 400044, China,, \\ E-mail:wangyongcq@126.com, \\ ${ }^{3}$ SMART Infrastructure Facility, University of Wollongong, \\ Northfields Ave, Wollongong, NSW 2522, Australia, \\ E-mail: \{jma,jiey\}@uow.edu.au
}

Received 24 June 2017

Accepted 12 February 2018

\begin{abstract}
E-commerce has grown exponentially in the past decade in global market. In China most E-commerce enterprises are small and medium-sized (SMEs). Compared to their large-sized counterparts, SMEs have to face many obstacles when extending their E-commerce businesses. In view of the complexity and periodicity of criteria in SMEs' development, the paper develop an evaluation method using complex fuzzy sets (CFS) to help them select appropriate development path. Then the paper focuses on a case study in Chongqing, China and compares the results with two other different methods (TOPSIS and COPRAS). The study indicates that the presented work can better handle uncertainty and periodicity in the evaluation process.
\end{abstract}

Keywords: SMEs, E-commerce, complexity fuzzy sets, TOPSIS, COPRAS, fuzzy methods

\section{Introduction}

E-commerce has grown exponentially in the past decade in global market. The benefits of Ecommerce are apparent not only for large companies but also for small and medium-sized enterprises (SMEs) ${ }^{11}$. In China, over 99\% of newly emerging enterprises are SMEs; and the number of SMEs who employ E-commerce exceeded 48 million ${ }^{35}$. According to "SMEs Export" (2012), 60\% of China's exports are contributed by SME exporters. SMEs have been playing key roles in Chinese economic development ${ }^{29}$. However, compared to large-sized enterprises, there exist many obstacles for Chinese SMEs to extend their E-commerce businesses.

Firstly, most researches regarding to SMEs and E-commerce just occur in an international domain 20. There has been little findings reported on SME E-commerce in developing countries ${ }^{10}$; and majority of the research, particularly those focused on developing countries, is merely qualitative (e.g. Refs. $9,21,27,30)$.

Secondly, although existing studies of E- 
commerce have examined factors such as user acceptance, consumer behaviour, E-commerce software, investment decision making factors in adopting E-commerce, selection of E-commerce sites by the consumer, the impact of innovation and pricing strategies $1,3,5,12,14,15,16,17,18,6,24,25,26,31,32$, few studies have investigated the magnitude of all these factors on E-commerce development in SMEs. Thus, elucidating the factors required for successful Ecommerce development path, particularly in the SME sector, is a worthwhile endeavour for both researchers and SME managers.

To overcome the above-mentioned research gaps, the objective of this study is to evaluate E-commerce development paths among managers/owners of SMEs in China. So this study focuses on different new development paths on SMEs E-commerce. Considering the uncertainties and periodicities in the evaluation, we choose the complex fuzzy set (CFS) as primary tool.

The reminder of the paper is organized as follows. Section 2 presents complex fuzzy sets and relevant operations on them. Section 3 presents a complex fuzzy set based evaluation method for the identified development paths, called the SME-C method. section 4 focuses on a case study in Chongqing, China and compares the results with two other different methods. Finally, Section 5 discusses the conclusions and future works.

\section{Complex fuzzy sets}

A complex fuzzy set (CFS) is an extension of a conventional fuzzy set (FS) by adopting complexvalued membership degrees to express and process the co-existing uncertainty and periodicity in many applications simultaneously, e.g. signal processing 23,22 . Roughly speaking, the significant difference between a CFS and an FS is the co-domain on which the membership degree is defined. The membership degree of a CFS is defined in the unit disc $D_{\mathbb{C}}=\{d ;\|d\| \leqslant 1\}$ on the complex plane; while it is the unit interval $[0,1]$ of the real numbers of an FS. Formally, a CFS $\widetilde{A}$ on a discourse $U$ is defined as

$$
\widetilde{A}=\left\{\left(u, \mu_{\widetilde{A}}(u)\right) ; u \in U, \mu_{\widetilde{A}}(u) \in D_{\mathbb{C}}\right\}
$$

where $\mu_{\widetilde{A}}(u)$ is conveniently expressed by the Polar coordinate as

$$
\mu_{\widetilde{A}}(u)=r_{u} e^{i \phi_{u}}
$$

and therefore $r_{u}$ and $\phi_{u}$ are called the modulus and the phase parts of a complex-valued membership degree respectively. Because of the introduction of complex-valued membership degrees, a CFS is capable of modelling real-world problems which involve both uncertainty and "approximately periodic" phenomena ${ }^{4}$.

The complex-valued membership degree used in the CFS theory enhances its capability of describing complicated uncertainties and enriches the theory of the conventional FS. However, an issue has raised naturally that a complex-valued membership degree is hard to be understood compared with the conventional real-valued membership degree. To clarify the necessity of a complex-valued membership degree, Ref. 28 presented a new interpretation of it from a complex fuzzy class which is embedded with the complex fuzzy set class operations.

Similar to conventional FS theory, operations are defined for CFSs. However, they are not intuitively understandable compared to those defined for conventional FSs. Refs. 22, 23 defined some settheoretical operations which are mainly defined on the modulus part of the complex-valued membership degrees rather than the phase part. Ref. 7 presented the concept of rotational invariance and defined a complex fuzzy logic system to address how to combine them closely. In order to deliver operations that better illustrate the phase part of a CFS, Ref. 37 defined the distance and $\delta$-similarity of two CFSs considering both the modulus and the phase parts. However, the modulus and phase are combined loosely. Research indicates that the main challenge is how to give an intuitional and readily understandable definition for the phase part of a CFS.

A Pythagorean fuzzy set (PFS) is a kind of extension of intuitionistic fuzzy set ${ }^{2}$ presented by Ref. 34 which is very similar to a CFS except that the membership degree is defined on the first-quarter of the unit disc of the complex plane. Ref. 33 discussed its applications in multi-criteria decision making. Furthermore, Ref. 8 discussed the relationships between CFSs and Pythagorean fuzzy sets. In below discus- 
sion of aggregation and partial order for CFSs, we borrowed some ideas from the Pythagorean fuzzy sets.

Ref. 34 defined the general forms of aggregations for the Pythagorean fuzzy sets; and the weighted quasi-power mean Agg is defined as

$$
\begin{aligned}
& \operatorname{Agg}\left(\left\{C\left(a_{i}, b_{i}\right)\right\}_{i=1}^{n},\left\{w_{i}\right\}_{i=1}^{n}\right) \\
& =\left(\left(\sum_{i=1}^{n} w_{i} a_{i}^{2}\right)^{1 / 2},\left(\sum_{i=1}^{n} w_{i} b_{i}^{2}\right)^{1 / 2}\right)
\end{aligned}
$$

where $C\left(a_{i}, b_{i}\right)$ is a Pythagorean membership satisfying $a_{i}, b_{i} \in[0,1]$ such that $a_{i}^{2}+b_{i}^{2} \leqslant 1, i=1, \ldots, n$; and $\sum_{i=1}^{n} w_{i}=1$. In Eq. (3), we note that

$$
\begin{aligned}
& \sum_{i=1}^{n} w_{i} a_{i}^{2} \leqslant \sum_{i=1}^{n} w_{i} a_{i} \\
& \sum_{i=1}^{n} w_{i} b_{i}^{2} \leqslant \sum_{i=1}^{n} w_{i} b_{i} .
\end{aligned}
$$

and the weighted vector sum of all $C\left(a_{i}, b_{i}\right) \mathrm{s}$ is

$$
\left(\sum_{i=1}^{n} w_{i} a_{i}, \sum_{i=1}^{n} w_{i} b_{i}\right)=\sum_{i=1}^{n} w_{i} C\left(a_{i}, b_{i}\right)
$$

If we can prove the weighted vector sum is in the unit disc $D_{\mathbb{C}}$, then we can use it as the aggregation of all $C\left(a_{i}, b_{i}\right)$ s. In fact, this can be proved by the Cauchy-Schwarz inequality. Inspired by this definition, we will define the weighted mean Agg on CFSs as

$$
\begin{aligned}
& \operatorname{Agg}\left(\left\{\widetilde{A}\left(a_{i}, b_{i}\right)\right\}_{i=1}^{n},\left\{w_{i}\right\}_{i=1}^{n}\right) \\
& =\left(\sum_{i=1}^{n} w_{i} a_{i}, \sum_{i=1}^{n} w_{i} b_{i}\right)
\end{aligned}
$$

where $a_{i}=r_{i}(u) \cos \left(\phi_{i}(u)\right)$, and $b_{i}=$ $r_{i}(u) \sin \left(\phi_{i}(u)\right)$. By $a_{i}^{2}+b_{i}^{2}=r_{i}^{2}(u) \leqslant 1$, we can prove that the right-hand of Eq. (6) falls in the unit disc of the complex plane and can be used as membership degree of a CFS.

After defining aggregation on CFS, another issue we need to solve is how to order (rank) the aggregated result. In literatures, we can easily find several different methods based on various initiatives. Yager and Abbasov ${ }^{34}$ ordered the aggregated result using a binary function $F$ from a Pythagorean membership degree to a value in $[0,1]$ :

$$
F(r, \theta)=\frac{1}{2}+r\left(\frac{1}{2}-\frac{2 \theta}{\pi}\right)
$$

where $C(a, b)$ is a Pythagorean membership grade, $r^{2}=a^{2}+b^{2}$ and $\theta=\arctan \left(\frac{b}{a}\right)$. Because the definition given is in the first quarter of the unit disc, we choose the following definition given in Ref. 19 to extend it to the whole unit disc: for any two CFSs $\widetilde{A}$ and $\widetilde{B}$,

$$
\widetilde{A} \leqslant \widetilde{B} \Longleftrightarrow r_{\widetilde{A}} \cos \left(\phi_{\widetilde{A}}\right) \leqslant r_{\widetilde{B}} \cos \left(\phi_{\widetilde{B}}\right) .
$$

\section{The SME-C method}

Using the discussion of aggregation and partial order for CFSs in Section 2, this secion proposes an evaluation method for SME development path which is named the SME-C method. Firstly, we describe the basic formalisation of the application; then outline the main steps of the SME-C method; and finally discuss details of each step.

\subsection{Formalisation of the application}

Given a set of $n$ candidate development paths (referred as alternatives $\left.a_{i}, i=1, \ldots, n\right)$, a set of $m$ criteria $\left(c_{j}, j=1, \ldots, m\right)$ and their corresponding weights $\left(w_{j}, j=1, \ldots, n\right)$, and an initial assessment matrix $X=\left(x_{i j}\right)_{n \times m}$, the utimate target of the application is to select the best candidate path(s) based on available information. This formalisation is a typical multi-criteria decision making configuration without any other specifications. In this paper, we require $x_{i j}$ to be a complex-valued membership degree of an underlying CFS $\widetilde{F}_{j}, j=1, \ldots, m$.

\subsection{Outline of the SME-C method}

The steps of the SME-C method are briefly listed below:

Step 1 Normalise initial assessment matrix $X$ to normalized assessment matrix $\bar{X}$;

Step 2 Generate weighted assessment matrix $\widehat{X}$ from $\bar{X}$; 
Step 3 Calculate aggregated assessment $S_{i}$ for each alternative;

Step 4 Rank $S_{i}$ s based on partial order given in Eq. (8).

\subsection{Normalisation of initial assessment matrix}

That normalising initial assessment matrix is commonly attribute to two practical purposes: 1) to make the comparisons between different criteria on a unified measurement scale; and 2) to make the following calculation or operation confined to specific properties. In the SME-C method, this step is optional when setting $x_{i j}$ to be a complex-valued membership degree. When the requirement is not satisfied, we need to normalise the initial assessment matrix $X$ to meet the aforementioned two purposes. Hence, we give two possible normalised methods, named type-I and type-II.

Definition 1. Let $\Delta_{I, j}=\sum_{i=1}^{n}\left\|\widetilde{x}_{i j}\right\|$, then the type-I normalisation of $\widetilde{x}_{i j}, i=1, \ldots, n$, gives

$$
\overline{\widetilde{x}}_{i j}=\frac{\widetilde{x}_{i j}}{\Delta_{I}}
$$

Definition 2. Let $\Delta_{I I, j}=\left\|\sum_{i=1}^{n} \widetilde{x}_{i j}\right\|$, then the type-II normalisation of $\widetilde{x}_{i j}, i=1, \ldots, n$, gives

$$
\overline{\widetilde{x}}_{i j}=\frac{\widetilde{x}_{i j}}{\Delta_{I I}}
$$

Following Definition 1 and Definition 2, we can show that under both normalisations, the vector sum $\sum_{i=1}^{n} \overline{\widetilde{x}}_{i j}$ of normalised assessments $\overline{\widetilde{x}}_{i j}, i=1, \ldots, n$, is in the unit disc $D_{\mathbb{C}}$.

\subsection{Generation of weighted assessment matrix}

This step aims at measuring the influence of each criterion $c_{j}$ by importing its corresponding weight $w_{j}$ into the normalised assessment matrix. For any $\overline{\widetilde{x}}_{i j}$, the weighted assessment is defined as $\widehat{\widetilde{x}}_{i j}=$ $w_{j} \overline{\widetilde{x}}_{i j}, i=1, \ldots, n, j=1, \ldots, m$. Obviously, $\widehat{\widetilde{x}}_{i j} \in D_{\mathbb{C}}$ and $\sum_{i=1}^{n} \widehat{\widetilde{x}}_{i j} \in D_{\mathbb{C}}$.

\subsection{Calculation and ranking of aggregated assessment}

By Eq. (6) and the fact that $\sum_{i=1}^{n} \widehat{\widetilde{x}}_{i j} \in D_{\mathbb{C}}$, we define the aggregated assessment $S_{i}=\sum_{i=1}^{n} \widehat{\widetilde{x}}_{i j}$. Once $S_{i}$ is obtained, we can rank them by the partial order given in Eq. (8). This is a straightforward step.

\section{Case study}

\subsection{Background}

Chongqing is the fourth municipality of China. It is historically a heavy and military industry base because of its remoteness in geography. With the acceleration of China's National Western Development Strategy, more and more new economy forms have been developed in the past two decades. Small and medium-sized E-commerce enterprises have rapidly emerged in last 10 years. However, due to the inland location and limited volumes of products and services, these enterprises face numerous constraints on e-commerce development. In this study, we choose one typical enterprise as illustrative example to solve its development path selection issue. There are three potential paths for the enterprise to choose from. Path 1 is Whole course Ecommerce model based on cloud computing. Path 2 is Location based service (LBS) model based on Ecommerce integrator (E-integrator). Path 3 is third party market mode based on information technology. To make sure which path is the best for the enterprise, a third-partied consultancy has identified seven criteria to measure which are Core Strategy Management $\left(c_{1}\right)$, Learning and Capability Development $\left(c_{2}\right)$,Information and Communication Technology (ICT, $c_{3}$ ), Convenience and Security of Data Information $\left(c_{4}\right)$, Cost $\left(c_{5}\right)$, Product and Service $\left(c_{6}\right)$, and Enterprise Stakeholders $\left(c_{7}\right)$.

Regarding to each individual criterion, the consultancy defined its optimisation direction and weights for each alternative path which are listed in Table 1. In these seven criterion, two are negative. For cost $\left(c_{5}\right)$ criterion, it is obvious that the less the cost, the better for the enterprise. For Enterprise stakeholders $\left(c_{7}\right)$, we argue for the small enterprise, 
the less the stakeholder, the easier for them to make new measures.

In these seven criterion, we argue that criteria $c_{2}$, $c_{5}$ and $c_{7}$ are periodic. When the enterprise face a new circumstance, people need spend more time to learn it. With the increase in learning time, people are more and more skilled and therefore improve significantly work efficiency and increase adaptability. Finally everyone will get used to it. When facing new circumstance again, the same process repeats. So we argue learning and capability development $\left(c_{2}\right)$ is a periodic criterion. According to the degree of learning difficulty, the paper define $c_{2}$ a $(0, \pi / 2)$ periodic criterion. In enterprise development, cost $\left(c_{5}\right)$ and stakeholder relationship $\left(c_{7}\right)$ are affected by many factors and often depict periodic features. Hence the paper defines them as periodic criteria and assigns a $(0, \pi)$ period.

With respected to the seven criteria, evaluations on the three paths are given (see Table 2) based on underlying CFSs of those criteria.

Table 2. Initial assessment matrix (“+” = positive, “-” = negative).

\begin{tabular}{c|c|rr|rr|rr}
\hline Criteria & Direction & $r_{1}$ & $\phi_{1}$ & $r_{2}$ & $\phi_{2}$ & $r_{3}$ & $\phi_{3}$ \\
\hline$c_{1}$ & + & 2 & 0 & 3 & 0 & 1 & 0 \\
$c_{2}$ & + & 1 & $0 \pi$ & 3 & $\pi / 3$ & 2 & $\pi / 6$ \\
$c_{3}$ & + & 1 & 0 & 2 & 0 & 3 & 0 \\
$c_{4}$ & + & 3 & 0 & 2 & 0 & 1 & 0 \\
$c_{5}$ & - & 3 & $\pi / 2$ & 2 & $3 \pi / 4$ & 1 & $\pi$ \\
$c_{6}$ & + & 3 & 0 & 2 & 0 & 1 & 0 \\
$c_{7}$ & - & 1 & $\pi / 2$ & 3 & $\pi / 3$ & 2 & $\pi / 6$ \\
\hline
\end{tabular}

In order to rank the potential paths, we apply the SME-C method. The ranking is conducted as follows:

\section{Step 1: Normalise assessment matrix $X$}

Let $X=\left(x_{i j}\right)_{7 \times 3}$ be the initial assessment matrix given in Table 3 .
Table 3. Initial assessment matrix in terms of Cardinal coordinate

\begin{tabular}{c|r|r|r}
\hline & \multicolumn{3}{|c}{ Cardinal coordinate } \\
\hline criteria & $a_{1}$ & $a_{2}$ & $a_{3}$ \\
\hline$c_{1}$ & $2+0 \mathrm{i}$ & $3.000+0.000 \mathrm{i}$ & $1.000+0.000 \mathrm{i}$ \\
$c_{2}$ & $1+0 \mathrm{i}$ & $1.527+2.582 \mathrm{i}$ & $1.731+1.002 \mathrm{i}$ \\
$c_{3}$ & $1+0 \mathrm{i}$ & $2.000+0.000 \mathrm{i}$ & $3.000+0.000 \mathrm{i}$ \\
$c_{4}$ & $3+0 \mathrm{i}$ & $2.000+0.000 \mathrm{i}$ & $1.000+0.000 \mathrm{i}$ \\
$c_{5}$ & $0+3 \mathrm{i}$ & $-1.414+1.414 \mathrm{i}$ & $-1.000+0.000 \mathrm{i}$ \\
$c_{6}$ & $3+0 \mathrm{i}$ & $2.000+0.000 \mathrm{i}$ & $1.000+0.000 \mathrm{i}$ \\
$c_{7}$ & $0+1 \mathrm{i}$ & $1.527+2.582 \mathrm{i}$ & $1.731+1.002 \mathrm{i}$ \\
\hline
\end{tabular}

As discussed above, we use the type-I normalisation for illustration purpose. For $j=1$,

$$
\Delta_{I, 1}=2+3+1=6 \text {. }
$$

Hence, the type-I normalisation result is $0.400+$ $0.000 i\left(a_{1}\right), 0.333+0.000 i\left(a_{2}\right), 0.267+0.000 i\left(a_{3}\right)$. Similarly, we can calculate the normalised assessments for other criteria.

$$
\begin{aligned}
& \left(\begin{array}{ccc}
0.333+0.000 i & 0.500+0.000 i & 0.167+0.000 i \\
0.167+0.000 i & 0.254+0.430 i & 0.289+0.167 i \\
0.167+0.000 i & 0.333+0.000 i & 0.500+0.000 i \\
0.500+0.000 i & 0.333+0.000 i & 0.167+0.000 i \\
0.000+0.500 i & -0.236+0.236 i & -0.167+0.000 i \\
0.500+0.000 i & 0.333+0.000 i & 0.167+0.000 i \\
0.000+0.167 i & 0.255+0.430 i & 0.289+0.167 i
\end{array}\right) \\
& \left(\begin{array}{ccc}
0.033+0.000 i & 0.050+0.000 i & 0.017+0.000 i \\
0.017+0.000 i & 0.025+0.043 i & 0.029+0.017 i \\
0.017+0.000 i & 0.033+0.000 i & 0.050+0.000 i \\
0.100+0.000 i & 0.067+0.000 i & 0.033+0.000 i \\
0.000+0.100 i & -0.047+0.047 i & -0.033+0.000 i \\
0.100+0.000 i & 0.067+0.000 i & 0.033+0.000 i \\
0.000+0.017 i & 0.025+0.043 i & 0.029+0.017 i
\end{array}\right)
\end{aligned}
$$

Step 3: Calculate aggregated assessment $S_{i}$

For each alternative, the aggregated assessment $S_{i}$ is calculated based on Eq. (6). Here, we can simply add the weighted assessments for each alternative; therefore, they are shown in Table 4. 
Table 1. Criteria for E-commerce development and predefined weights.

\begin{tabular}{llrl}
\hline Criterion & Description & weight & Direction \\
\hline$c_{1}$ & core strategy development & 0.1 & positive \\
$c_{2}$ & learning and capability development & 0.1 & positive \\
$c_{3}$ & ICT & 0.1 & positive \\
$c_{4}$ & convenience and security for data information & 0.2 & positive \\
$c_{5}$ & cost & 0.2 & negative \\
$c_{6}$ & product and service & 0.2 & positive \\
$c_{7}$ & enterprise stakeholders & 0.1 & negative \\
\hline
\end{tabular}

Table 4. Aggregated assessment for each alternative path.

\begin{tabular}{ccc}
\hline$S_{1}$ & $S_{2}$ & $S_{3}$ \\
\hline $0.267+0.117 \mathrm{i}$ & $0.220+0.133 \mathrm{i}$ & $0.158+0.033 \mathrm{i}$ \\
\hline
\end{tabular}

Step 4: Rank alternative paths

Based on the aggregated assessments and Eq. (8), path $a_{1}$ is the best path and then followed by $a_{2}$ and $a_{3}$.

\subsection{Discussions and comparisons}

In this section, we will compare the presented SME$\mathrm{C}$ method with other two popular multi-criteria decision methods, i.e. the COPRAS and the TOPSIS. We choose these two methods because they have similar processing steps and they need to identify positive and negative decision direction which we can use the phase part of a complex-valued membership degree to imitate.

The COPRAS method is a widely-used multicriteria decision making technique, which contains three main steps ${ }^{36}$ : (1) normalises initial assessments regarding to each individual evaluation criterion; (2) calculates two optimisation indexes for each alternative based on criteria' optimisation directions (decision directions); and (3) ranks alternatives based on an overall index calculated from optimisation indexes. These steps are briefly described below.

Suppose $X$ is the initial assessment matrix

$$
X=\left(\begin{array}{ccc}
x_{11} & \cdots & x_{1 m} \\
\vdots & \ddots & \vdots \\
x_{n 1} & \cdots & x_{n m}
\end{array}\right)
$$

then the normalised assessment $\bar{x}_{i j}$ is given as

$$
\bar{x}_{i j}=\frac{x_{i j}}{\sum_{k=1}^{n} x_{k j}},
$$

and the weighted assessment $\widehat{x}_{i j}$ ) is

$$
\widehat{x}_{i j}=\bar{x}_{i j} \cdot w_{j},
$$

for $i=1, \ldots, n, j=1, \ldots, m$.

After getting the weighted assessment matrix $\widehat{X}$, the COPRAS needs to calculate optimisation indexes determined by the preferable optimisation direction of each criterion. A $c_{j}$ is associated with one of two preferable optimisation directions, i.e., positive (the bigger the better, a.k.a. "optimisation direction is maximisation") or negative (the smaller the better, a.k.a. "optimisation direction is minimisation"). Without loss of generality, let $C^{+}$be the set of criteria with positive optimisation direction and $C^{-}$be the set of criteria with negative optimisation direction, then for each alternative $a_{i} \in A$ two optimisation indexes corresponding to $C^{+}$and $C^{-}$respectively can be calculated as

$$
S_{i}^{+}=\sum_{c_{j} \in C^{+}} \widehat{x}_{i j}, \quad S_{i}^{-}=\sum_{c_{j} \in C^{-}} \widehat{x}_{i j}, \quad i=1,2, \ldots, n
$$

Using these two optimisation indexes, an overall ranking index $Q_{i}$ is therefore calculated for each alternative $a_{i}$ :

$$
Q_{i}=S_{i}^{+}+\frac{\sum_{k=1}^{n} S_{k}^{-}}{S_{i}^{-} \sum_{k=1}^{n} \frac{1}{S_{k}^{-}}}, \quad i=1,2, \ldots, n
$$


Finally, $Q_{i},(i=1,2, \ldots, n)$ is used to rank alternatives. A higher $Q_{i}$ means a better assessment on $a_{i}$.

By using the COPRAS method, we get a similar ranking with the following reference indexes (Table 5).

Table 5. Ranking result by COPRAS method.

\begin{tabular}{ccccc}
\hline & $S^{-}$ & $S^{+}$ & $Q$ & rank \\
\hline$a_{1}$ & 0.117 & 0.267 & 0.347 & 1 \\
$a_{2}$ & 0.117 & 0.267 & 0.347 & 1 \\
$a_{3}$ & 0.067 & 0.167 & 0.307 & 3 \\
\hline
\end{tabular}

The TOPSIS (Technique for Order of Preference by Similarity to Ideal Solution) may be the most-used multi-criteria decision making method, which has many different extensions. TOPSIS has two main difference compared with the COPRAS method. The first one is the normalisation method used. TOPSIS adopts the vector-based normalisation, i.e.

$$
\bar{x}_{i j}=\frac{x_{i j}}{\sqrt{\sum_{i=1}^{n} x_{i j}^{2}}}
$$

This normalisation is exactly the Type-II normalisation in the presented SME-C method. The second difference is how to get the ranking index. By TOPSIS, the index is calculated as

$$
Q_{i}=\frac{d_{i w}}{d_{i w}+d_{i b}}
$$

where

$$
d_{i w}=\sqrt{\sum_{j=1}^{n}\left(\widehat{x}_{i j}-\widehat{x}_{w j}\right)^{2}}, \quad d_{i b}=\sqrt{\sum_{j=1}^{n}\left(\widehat{x}_{i j}-\widehat{x}_{b j}\right)^{2}}
$$

$\widehat{x}_{w j}=\left\{\max _{c_{j} \in C_{-}} \widehat{x}_{i j}, \min _{c_{j} \in C_{+}} \widehat{x}_{i j}\right\}, \quad \widehat{x}_{b j}=\left\{\min _{c_{j} \in C_{-}} \widehat{x}_{i j}, \max _{c_{j} \in C_{+}} \widehat{x}_{i j}\right\}$.

By using the TOPSIS method, we get a similar ranking with the following reference indexes (Table 6).

Table 6. Ranking result by TOPSIS method.

\begin{tabular}{ccc}
\hline & $Q_{i}$ & rank \\
\hline$a_{1}$ & 0.549 & 1 \\
$a_{2}$ & 0.526 & 2 \\
$a_{3}$ & 0.432 & 3 \\
\hline
\end{tabular}

Our previous experiments indicate, the TOPSIS and the COPRAS methods can produce similar ranking in most situations. In this example, we get the same ranking using three different methods.

\section{Conclusion}

\subsection{Summary}

The purpose of this paper was to contribute towards an analytical understanding of E-commerce development path in SMEs. We summarised three development paths for SMEs, identified seven different criteria and evaluated them with CFS. Finally we compared the results with other two popular methods (COPRAS and TOPSIS).

\subsection{Limitations and Suggestions for further study}

It is inevitable that researchers deal with some limitations in their studies and the present study is no exception. These limitations set stage for future research.

Firstly, this study used a limited number of experts from a third-party consultancy. Future research may repeat this method using multiple experts to justify the validity of the study.

Secondly, it should be mentioned that understanding the barriers and drivers of SME-C implementation helps E-commerce to introduce costly effective practices. So, further research will attempt to focus on this issue.

Thirdly, this research has explored only one case study in a small E-commerce enterprise. Hence conclusions may not generally suit various companies and industries. Future works should conduct research related to investigation on E-commerce practices and performances in different sectors.

Finally, identifying weights and decision direction is an important issue in the evaluation of enterprise development strategy. In the presented work, we used a predefined weights and direction from the consultancy. We noted that this may include too much personal or subjective opinions and we could improve it by using pairwise comparison like that used widely in fuzzy AHP research area ${ }^{13}$. This 
may require more discussion about how to efficient calculation on CFS. We will work on this in future work.

\section{Acknowledgement}

This work was supported by Major Cultivating Project on Chongqing University of Arts and Science under Grant, 2016 Post-doctoral Research Funding Project in Chongqing under Grant (number xm2016094),2017 key Topics of Chongqing Education Science Planning Project under Grant and National Natural Science Foundation of China under Grant (number 71672015).

\section{Conflict of interest}

The authors declare that there is no conflict of interests regarding the publication of this article.

\section{References}

1. Niklas Aldin and Fredrik Stahre. Electronic commerce, marketing channels and logistics platformsa wholesaler perspective. European Journal of Operational Research, 144(2):270 - 279, 2003.

2. Krassimir T. Atanassov. Intuitionistic fuzzy sets. Fuzzy Sets and Systems, 20(1):87 - 96, 1986.

3. Amit Basu and Steve Muylle. Online support for commerce processes by web retailers. Decision Support Systems, 34(4):379 - 395, 2003.

4. Zhifei Chen, Sara Aghakhani, James Man, and Scott Dick. ANCFIS: a neurofuzzy architecture employing complex fuzzy sets. IEEE Transactions on Fuzzy Systems, 19(2):305-322, 2011.

5. Se-Hak Chun and Jae-Cheol Kim. Pricing strategies in b2c electronic commerce: analytical and empirical approaches. Decision Support Systems, 40(2):375 388, 2005.

6. Dirk Van den Poel and Wouter Buckinx. Predicting online-purchasing behaviour. European Journal of Operational Research, 166(2):557 - 575, 2005.

7. Scott Dick. Toward complex fuzzy logic. IEEE Transactions on Fuzzy Systems, 13(3):405-414, 2005.

8. Scott Dick, Ronald R. Yager, and Omolbanin Yazdanbakhsh. On Pythagorean and complex fuzzy set operations. IEEE Transactions on Fuzzy Systems, 24(5): 1009 - 1021, 2016.

9. Ali F. Farhoomand, Virpi Kristiina Tuunainen, and Lester W. Yee. Barriers to global electronic commerce: A cross-country study of hong kong and fin- land. Journal of Organizational Computing and Electronic Commerce, 10(1):23-48, 2000.

10. Morteza Ghobakhloo, Tang Sai Hong, and Craig Standing. B2b e-commerce success among small and medium-sized enterprises: A business network perspective. 27(1):1-32, 2015.

11. Elizabeth E. Grandon and J.Michael Pearson. Electronic commerce adoption: an empirical study of small and medium us businesses. Information \& Management, 42(1):197 - 216, 2004.

12. Shi-Ming Huang, Yu-Chung Hung, and David C. Yen. A study on decision factors in adopting an online stock trading system by brokers in taiwan. Decision Support Systems, 40(2):315 - 328, 2005.

13. Cengiz Kahraman, Ufuk Cebeci, and Ziya Ulukan. Multi-criteria supplier selection using fuzzy ahp. Logistics Information Management, 16(6):382-394, 2003.

14. $\mathrm{Li} \mathrm{Li}$ and Dimitrios Buhalis. E-commerce in china: The case of travel. International Journal of Information Management, 26(2):153 - 166, 2006.

15. Chechen Liao, Pui-Lai To, and Meng-Lin Shih. Website practices: A comparison between the top 1000 companies in the us and taiwan. International Journal of Information Management, 26(3):196 - 211, 2006.

16. Jyh-Horng Lin and Rosemary Jou. Financial ecommerce under capital regulation and deposit insurance. International Review of Economics \& Finance, 14(2):115 - 128, 2005.

17. Chang Liu, Jack T. Marchewka, June Lu, and ChunSheng Yu. Beyond concerna privacy-trust-behavioral intention model of electronic commerce. Information \& Management, 42(2):289 - 304, 2005.

18. Pin Luarn and Hsin-Hui Lin. Toward an understanding of the behavioral intention to use mobile banking. Computers in Human Behavior, 21(6):873 - 891, 2005.

19. Jun Ma, Guangquan Zhang, and Jie Lu. A method for multiple periodic factor prediction problems using complex fuzzy sets. IEEE Transactions on Fuzzy Systems, 20(1), 2012. 32-45.

20. Suzanne Altobello Nasco, Elizabeth Grandn Toledo, and Peter P. Mykytyn. Predicting electronic commerce adoption in chilean smes. Journal of Business Research, 61(6):697 - 705, 2008. Strategic Management in Latin America.

21. Simpson Poon and Paula M.C Swatman. An exploratory study of small business internet commerce issues. Information \& Management, 35(1):9 - 18, 1999.

22. Daniel Ramot, Menahem Friedman, Gideon Langholz, and Abraham Kandel. Complex fuzzy logic. IEEE Transactions on Fuzzy Systems, 11(4):450-461, 2003.

23. Daniel Ramot, Ron Milo, Menahem Friedman, and 
Abraham Kandel. Complex fuzzy sets. IEEE Transactions on Fuzzy Systems, 10(2):171-186, 2002.

24. Jose L. Salmeron and Jos M. Hurtado. Modelling the reasons to establish b2c in the fashion industry. Technovation, 26(7):865 - 872, 2006.

25. Joseph Sarkis and Srinivas Talluri. Evaluating and selecting e-commerce software and communication systems for a supply chain. European Journal of Operational Research, 159(2):318 - 329, 2004. Supply Chain Management: Theory and Applications.

26. Hung-Pin Shih. An empirical study on predicting user acceptance of e-shopping on the web. Information \& Management, 41(3):351 - 368, 2004.

27. Antonis C. Stylianou, Stephanie S. Robbins, and Pamela Jackson. Perceptions and attitudes about e-commerce development in china: An exploratory study. 11(2):31-47, 2003.

28. Dan E. Tamir, Naphtali D. Rishe, and Abraham Kandel. Complex fuzzy sets and complex fuzzy logic an overview of theory and applications. In Dan E. Tamir, Naphtali D. Rishe, and Abraham Kandel, editors, Fifty Years of Fuzzy Logics and Its Applications, volume 326 of Studies in Fuzziness and Soft Computing, pages 661-681. Springer, 2015.

29. Yehua Dennis Wei, Wangming Li, and Chunbin Wang. Restructuring industrial districts, scaling up regional development: a study of the wenzhou model, china. Economic Geography, 83(4):421-444, 2007.

30. William Wresch. Initial e-commerce efforts in nine least developed countries: A review of national infrastructure, business approaches, and product selection.
11(2):67-78, 2003.

31. Jen-Her $\mathrm{Wu}$ and Tzyh-Lih Hisa. Analysis of ecommerce innovation and impact: a hypercube model. Electronic Commerce Research and Applications, 3(4):389 - 404, 2004.

32. Jen-Her Wu and Yu-Min Wang. Development of a tool for selecting mobile shopping site: A customer perspective. Electronic Commerce Research and Applications, 5(3):192 - 200, 2006. Mobile technology and services.

33. Ronald R. Yager. Pythagorean membership grades in multicriteria decision making. IEEE Transactions on Fuzzy Systems, 22(4):958-965, 2014.

34. Ronald R. Yager and Ali M. Abbasov. Pythagorean membership grades, complex numbers, and decision making. International Journal of Intelligent Systems, 28:436 - 452, 2013.

35. Jia Yu and Jun Ni. Development strategies for SME e-commerce based on cloud computing. In $2013 \mathrm{Sev}$ enth International Conference on Internet Computing for Engineering and Science, pages 1-8, 2013.

36. Edmundas Kazimieras Zavadskas, Arturas Kaklauskas, Zenonas Turskis, and Jolanta Tamošaitiene. Multi-attribute decision-making model by applying grey numbers. Informatica, 20(2):305-320, 2009.

37. Guangquan Zhang, Tharam Signh Dillon, Kaiyuan Cai, Jun Ma, and Jie Lu. Operation properties and $\delta$-equalities of complex fuzzy sets. International Journal of Approximate Reasoning, 50(8):1227-1249, 2009. 